\title{
Modeling of Electron Kinetics in $\mathrm{BF}_{3}$
}

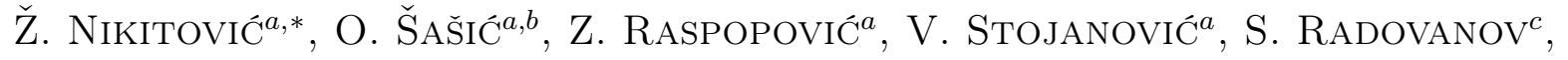 \\ M. MOZETIC̈ $\check{\check{C}}^{d}$ AND U. CVElBAR ${ }^{d}$ \\ ${ }^{a}$ Institute of Physics, POB 68, 11080 Belgrade, Serbia \\ ${ }^{b}$ Faculty of Transport and Traffic Engineering, University of Belgrade, Belgrade, Serbia \\ ${ }^{c}$ Varian Semiconductor Equipment Associates, 35 Dory Road, GL-17, Gloucester, MA01930 USA \\ ${ }^{d}$ Jozef Stefan Institute, Jamova cesta 39, 1000 Ljubljana, Slovenia
}

\begin{abstract}
In this paper we used the available data for the electron impact scattering cross sections $\mathrm{BF}_{3}$ to calculate the transport coefficients for electrons. Monte Carlo simulation was used to perform calculations of the transport coefficients as well as the rate coefficients in DC electric fields, crossed electric and magnetic DC and RF fields.
\end{abstract}

PACS numbers: 51.10.+y, 52.20.Fs, 52.65.Pp

\section{Introduction}

The fluid models are based on the transport data and on the cross section data normalized against the transport data $[1,2]$. Recent advances in the ion implantation include employing pulsed RF and DC afterglow who may be both modeled by swarm like or fluid models [3]. Operation of these devices requires further improvements and fine tuning and for that purpose detailed modeling is required. Recently, it was found that a number of kinetic phenomena exist that may affect these plasmas and could be explained at the level of kinetic theory or Monte Carlo simulation (MCS) $[4,5]$. Having in mind the complexity of these phenomena we have calculated transport coefficients on the basis of the existing set of cross sections [6]. Some of the preliminary data along similar lines were presented in [7]. One should be aware that in determination of the cross sections on the basis of the small amount of the existing transport data Biagi [6] has calculated some of the basic transport coefficients that are shown here but those are not generally available although may be easily reproduced.

Here we extend the modeling to analyze the magnitude of the effects such as non-conservative transport, negative differential conductivity (NDC) and transport in time varying and in crossed electric and magnetic fields. These data may be employed in the fluid modeling, as guidance for kinetic models and possible experiments.

\section{Numerical method}

Calculations were performed by using Monte Carlo codes for electron transport involving null collision

* corresponding author; e-mail: zeljka@ipb.ac.rs method or time integration technique. The code and the technique have been verified against the basic swarm benchmarks data $[8,9]$ and were shown to be in excellent agreement with other competing techniques for studies of electron kinetics.

We also consider non-conservative transport in the presence of ionization and/or attachment. For example the distinction should be made between the so called bulk $(b)$ and flux $(f)$ transport properties such as drift velocity $[8,9]$ :

$$
\boldsymbol{v}_{b}=\frac{\mathrm{d}}{\mathrm{d} t} \sum_{i=1}^{n} \boldsymbol{r}_{i} ; \quad \boldsymbol{v}_{f}=\sum_{i=1}^{n} \boldsymbol{v}_{i},
$$

which are identical in the conservative (no number changing collisions) case but differ appreciably with electron number changing collisions being significant. The null collision technique has been applied to model either an infinite swarm or the basic steady state Townsend experiment where spatial profiles of absolute emission (excitation) have been measured to provide the data on excitation coefficients, non-equilibrium processes (such as equilibration distance), secondary electron yields at the cathode surface and back diffusion [10].

Monte Carlo simulation is initiated for 500000 electrons with Maxwellian electron energy distribution function with mean energy of $1 \mathrm{eV}$. Gas number density was $3.54 \times 10^{22} \mathrm{~m}^{-3}$. Either only electric field or crossed electric and magnetic fields were applied. Calculations were made for DC and RF fields and in the latter case phase between electric and magnetic field was $\pi / 2$.

The basic set of data was taken compiled by S. Biagi [6]. The complete set of cross sections is shown in Fig. 1.

\section{Electron transport data in DC fields}

The test of cross sections for low energies was performed by comparing the calculated and experimental 


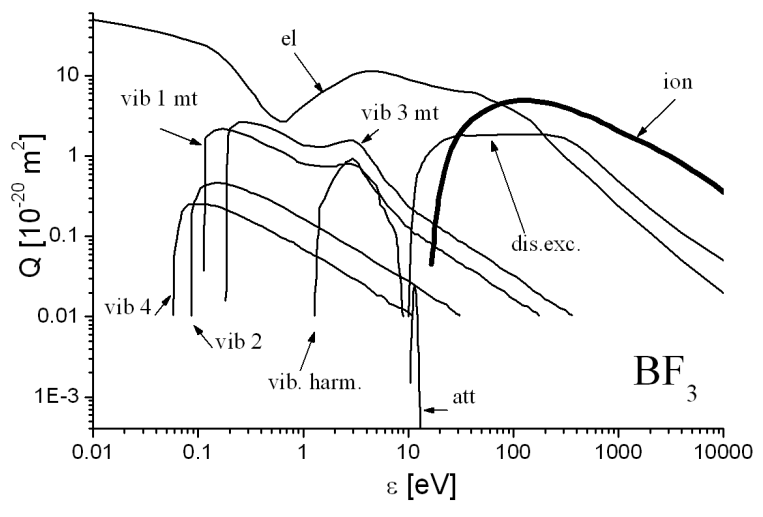

Fig. 1. Cross section set for electron $\mathrm{BF}_{3}$ collisions [1]. Elastic (el), ionization (ion), electron attachment (att), vibrational excitation (vib 1, vib 2, vib $3 \mathrm{mt}$, vib 4, vib. harm.), and dissociative excitation (dis.exc).

drift velocities and characteristic energies $\left(e D_{\mathrm{T}} / \mu\right.$, where $D_{\mathrm{T}}$ is the transverse diffusion coefficient and $\mu$ is mobility). For this purpose we have used both two term approximation and Monte Carlo simulations. At higher energies we compared the ionization rate, while assuming that the ionization cross section is very accurately determined and therefore should not be modified further. This allowed us to renormalize the set of electronic excitation cross sections. The transport coefficients in DC electric fields are shown in Fig. 2.

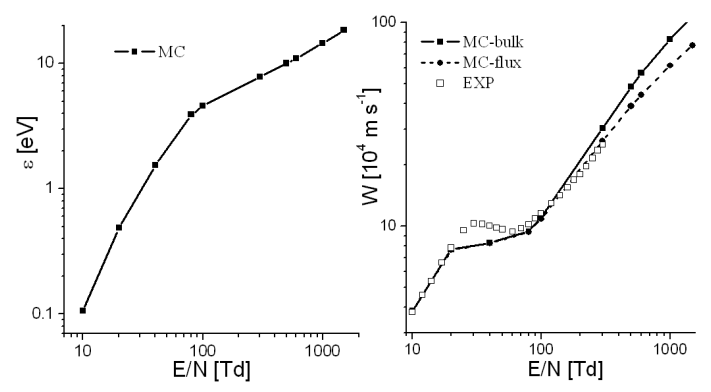

Fig. 2. Mean energy and drift velocity as a function $E / N$ for electrons in $\mathrm{BF}_{3}$. We denote the data from [11] by EXP.

The most striking effect in the $E / N$ dependence of drift velocity is the negative differential conductivity (NDC) in experimental data [11]. Even though the calculated drift velocity does not show the NDC, the shape of the cross sections is such that it supports the NDC so the drift velocity has a broad plateau from $20 \mathrm{Td}$ to $80 \mathrm{Td}$. Achieving a better fit would involve an increase in the vibrational cross section, or an increase in the decay of the peak of vibrational cross section, possibly augmented by a faster increase of the momentum transfer in the same energy range [12].

The diffusion is very anisotropic with $D_{\mathrm{T}} / D_{\mathrm{L}}$ ratio of up to 4 . One may conclude that both this anisotropy
$[13,1]$ and NDC may be associated with the Ramsauer Townsend minimum for NDC with the large vibrational cross sections.

We have obtained a complete set of collision rate coefficients required for continuum models [7] and some of the calculated data for $\mathrm{BF}_{3}$ given in Fig. 3. In that figure
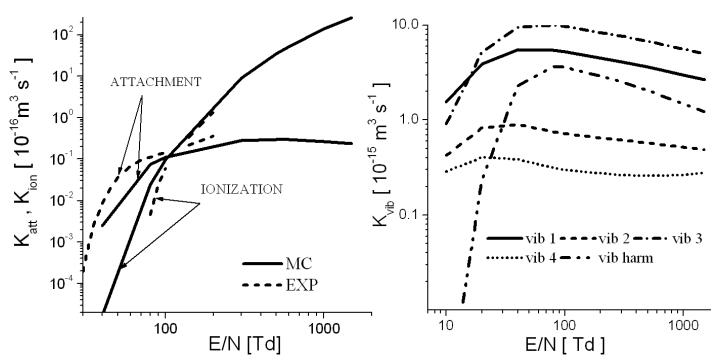

Fig. 3. Rate coefficients for electron $\mathrm{BF}_{3}$ collisions as a function of the local field normalized to the gas number density, a complete set based on cross sections from Fig. 1. EXP data are calculated [14] by using data of [11].

we compare calculated electron attachment and ionization coefficients to the experimental data obtained by the pulsed Townsend experimental technique [11]. While the agreement is not particularly good for attachment near the thresholds it is still sufficient for plasma modeling especially when $\mathrm{BF}_{3}$ is present in gas mixtures in a small abundance. We did not make any further adjustments of the cross sections since one would need more input from both binary collision theory/experiment or transport measurements. We also indicate that adjustment of the attachment cross section would have to be based on a further knowledge of the shape of attachment cross section. It could also stem from adjustment of energy controlling vibrational excitation cross sections.
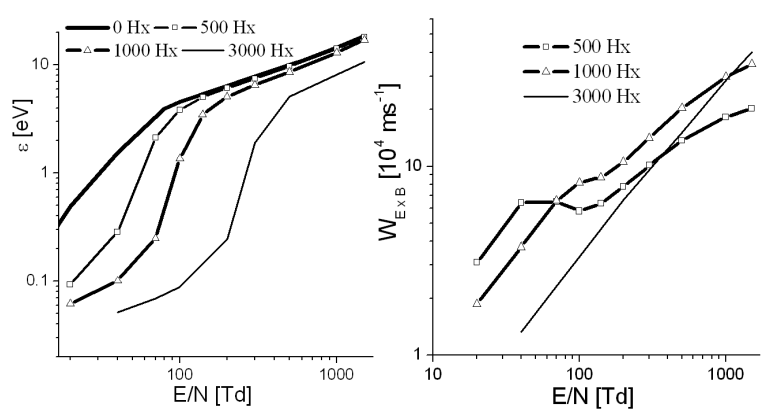

Fig. 4. Mean energy and drift velocity as a function $E / N$ for various $B / N$.

We, however, continue with analysis of how electrons in $\mathrm{BF}_{3}$ behave under different conditions that may lead to complex kinetic behaviour. The basic phenomenology of electron motion in DC ExB fields can be described in terms of $\omega_{\mathrm{c}} / \omega_{\mathrm{k}}$ where $\omega_{\mathrm{c}}$ is the cyclotron frequency and $\omega_{\mathrm{k}}$ is the collision frequency [15]. In Fig. 4.we show mean energy and perpendicular drift velocity as a function of 
$E / N$ for $B / N$ at 500,1000 and $3000 \mathrm{Hx}$. We observe three distinct regions of transport as $E / N$ increases. The first region is associated with slow increases in the mean energy owing to intensive energy losses arising from the vibrational excitation. The second region is that of a rapid rise of mean energy, and it develops when electrons gain sufficient energy to overcome the losses due to vibrational excitation. It usually coincides with the negative differential conductivity (NDC) region of the drift velocity which is observed for $\operatorname{ExB}$ component of the drift velocity. Finally, there is another region of slow increase in the mean energy as the electronic excitations become important in the controlling the energy of the swarm. In general, the application of magnetic field perpendicular to an electric field decreases the swarm mean energy for a given $E / N$. As a result, as $B / N$ increases, the mean energy curves apparently move to the right. In addition, at an increased $B / N$ there is a fastest variation in the mean energy in the range between 40 and $500 \mathrm{Td}$ where plasma chemistry may be modified significantly by changing the magnitude of the external magnetic field.

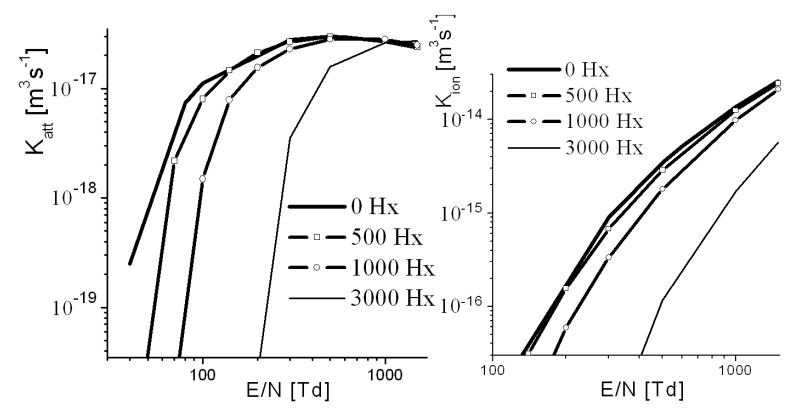

Fig. 5. Rate coefficients for electron $\mathrm{BF}_{3}$ collisions as a function of the local field normalized to the gas number density, a complete set based on cross sections from Fig. 1.

In Fig. 5. we show the ionization and attachment rate coefficients as a function of $E / N$ for various $B / N$. As expected, the ionization rate coefficient increases with $E / N$ and decreases with $B / N$. The attachment rate coefficient has a similar but not identical dependence on $E / N$ and $B / N$, showing stronger dependence on the magnetic field.

\section{Electron transport in $E(t) \times B(t)$ fields}

The Application of inductively coupled plasmas (ICP) and magnetically enhanced reactive ion etching raises the need to provide sets of data for different DC and RF combined magnetic and electric fields. In addition application of magnetic fields to confine/control the plasma may be employed in source plasmas for the ion implantation. In this paper we have performed calculations for perpendicular electric and magnetic RF fields. Hence the labels for axes: $E, B$ and $E x B$ are with phase difference of $\pi / 2$. Our goal was to study effect of frequency of applied fields, amplitude of reduced electric fields and amplitude of reduced magnetic fields in order to elucidate the possible complex kinetic behavior that may need kinetic explanation in the modeling and may also be employed to achieve desired goals.

The results of simulation are averaged over many periods of the fields and presented over a single period (regardless of the frequency). Time variation of the drift velocity as a function of magnetic field $(B / N)$ at $100 \mathrm{MHz}$ and pressure of 1 Torr) is shown in Fig. 6 . For $\pi / 2$ phase
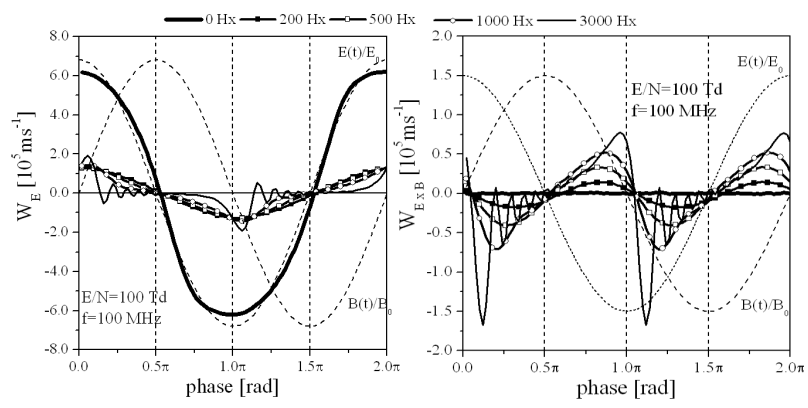

Fig. 6. Drift velocity for electrons in $\mathrm{BF}_{3}$ : a) in $\boldsymbol{E}$ direction and b) in $\boldsymbol{E} \times \boldsymbol{B}$ direction.

difference between electric and magnetic field, the longitudinal drift velocity is strongly suppressed by magnetic field while phase remains almost unchanged. In the presence of magnetic field both longitudinal and transverse drift velocities are of the same order of magnitude while in the opposite phases. Very high magnetic field introduces oscillations at frequency higher than driving frequency. These oscillations of both drift velocities which occur when cyclotron frequency exceeds collision frequency are coupled by phase.

\section{Conclusion}

The presently derived set of data provides a basis for a complete plasma model for plasma sources for ion implantation by using $\mathrm{BF}_{3}$ containing plasmas. Such data are the foundation for development of computer aided design of plasma devices $[1,16]$ and should include: electron scattering cross sections, DC electron transport data for $E$ and $E x B$ fields, ion-molecule reactions and excited state collision data.

Some of our Monte Carlo codes involve complete description of ions, fast neutrals and other particles that require kinetic description [17]. This could prove to be essential in direct modeling of extraction of ion beams or even formation of fast neutral beams from very low pressure plasmas [18].

\section{Acknowledgments}

This work was carried out with the support by the MNRS under grant No. 141025 and by joint funding from Serbian Ministry and Slovenian Ministry for High Education, Science and Technology (ARRS) grant BI-SRB-SLO-10-11 No. 1. Ž. Nikitović expresses special thanks to Slovenian Fund Ad-Futura for postdoc grant. 


\section{References}

[1] T. Makabe, Z.Lj. Petrović, Plasma Electronics New York: Taylor and Francis, (2006); Z.Lj. Petrović, S. Dujko, D. Marić, G. Malović, Ž. Nikitović, O. Šašić, J. Jovanović, V. Stojanović, M. Radmilović-Ra đenović unpublished J. Phys. D, Appl. Phys. (2009).

[2] R.E. Robson, R.D. White, Z.Lj. Petrović, Rev. Mod. Phys. 77, 1303 (2005).

[3] S. Radovanov, L. Godet, R. Dorai, Z. Fang, B.W. Koo, C. Cardinaud, G. Cartry, D. Lenoble, A. Grouillet, J. Appl. Phys. 98, 113307 (2005); L. Godet, Z. Fang, S. Walther, S. Radovanov, E. Arevalo, J. Scheuer, G. Cartry, C. Cardinaud, F. Lallement, D. Lenoble, JVST B 24, 2391 (2006).

[4] S. Dujko, R.D. White, Z.Lj. Petrović, J. Phys. D, Appl. Phys. 41, 245205 (2008).

[5] S. Dujko, R.D. White, K.F. Ness, Z.Lj. Petrović, R.E. Robson, J. Phys. D, Appl. Phys. 39, 4788 (2006), Z.Lj. Petrović, M. Šuvakov, Ž. Nikitović, S. Dujko, O. Šašić, J. Jovanović, G. Malović, V. Stojanović, Plasma Sources Sci. Technol. 16, S1 (2007).

[6] S. Biagi, private communication (2005).

[7] O. Šašić, Z.Lj. Petrović, Z. Raspopović, L. Godet, S. Radovanov, 58th Annual Gaseous Electronics Conference, San Jose, California, October 16-20, 2005, Bul. Am. Phys. Soc. 50, 28 UH2 4 (2005).
[8] Z. Raspopović, S. Sakadžić, Z.Lj. Petrović, T. Makabe, J. Phys. D, Appl. Phys. 33, 1298 (2000).

[9] Z.Lj. Petrović, V.D. Stojanović, JVSTA 16, 329 (1998).

[10] M. Radmilović, Z.Lj. Petrović, EPJ AP 11, 35 (2000).

[11] S.R. Hunter, J.G. Carter, L.G. Christophorou, J. Appl. Phys. 65, 1858 (1989).

[12] Z.Lj. Petrović, R.W. Crompton, G.N. Haddad, Aust. J. Phys. 3723 (1984); S.B. Vrhovac, Z.Lj. Petrović, Phys. Rev. E 53, 4012 (1996).

[13] T. Shimada, Y. Nakamura, Z.Lj. Petrović, T. Makabe, J. Phys. D 36, 1936 (2003).

[14] B.M. Jelenković, A.V. Phelps, Phys. Rev. A 36, 5310 (1987).

[15] S. Dujko, Z.M. Raspopović, Z.Lj. Petrović, J. Phys. D, Appl. Phys. 38, 2952 (2005).

[16] S. Rauf, M.J. Kushner, J. Appl. Phys. 85, 3460 (1999).

[17] V.D. Stojanović, Z.Lj. Petrović, J. Phys. D, Appl. Phys. 31, 834 (1998); Z.Lj. Petrović, A.V. Phelps, Phys. Rev. E 80, 016408 (2009).

[18] K. Endo, S. Noda, T. Ozaki, S. Samukawa, M. Masahara, Y. Liu, K. Ishii, H. Takashima, E. Sugimata, T. Matsukawa, H. Yamauchi, Y. Ishikawa, E. Suzuki, Jpn. J. Appl. Phys. 45, 5513 (2006). 